\title{
Combination of alpha lipoic acid and gabapentin, its efficacy in the treatment of Burning Mouth Syndrome: A randomized, double-blind, placebo controlled trial
}

\author{
Edgardo López-D’alessandro ${ }^{1}$, Livia Escovich ${ }^{2}$
}

\author{
${ }^{1}$ Master of Oral Medicine, Teacher researcher. Oral Medicine and Pharmacology Chairs. Faculty of Dentistry. National Univer- \\ sity of Rosario. Argentina \\ ${ }^{2}$ Professor of Oral Medicine. Director of the Master of Oral Medicine, Faculty of Dentistry. National University of Rosario. \\ Argentina
}

Correspondence:

San Luis 4303-Rosario,

Santa Fe, Argentina,

Post-code: 2000

iaoodontologia@hotmail.com

Received: 06/03/2010

Accepted: $31 / 05 / 2010$

López-D’alessandro E, Escovich L. Combination of alpha lipoic acid and gabapentin, its efficacy in the treatment of Burning Mouth Syndrome: A randomized, double-blind, placebo controlled trial. Med Oral Patol Oral Cir Bucal. 2011 Aug 1;16 (5):e635-40.

http://www.medicinaoral.com/medoralfree01/v16i5/medoralv16i5p635.pdf

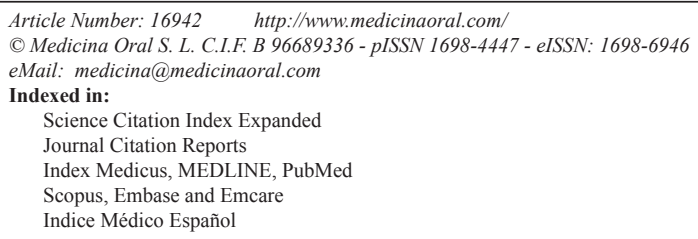

\begin{abstract}
Burning Mouth Syndrome (BMS) is a disease that manifests as burning in the tongue or in any area of the oral mucosa, in the absence of clinically verifiable injuries.

Objectives: To verify the efficacy of alpha lipoic acid (ALA) and gabapentin (GABA), used individually and jointly, to reduce the burning in patients with burning mouth and establish a drug therapy for the BMS.

Study Design: During April and May 2008, we conducted a randomized, double-blind, placebo-controlled trial in the Department of Clinical Stomatology, Faculty of Dentistry, Rosario, Argentina. The gathering of patients was between those ones with BMS who were treated in our service between March 2003 and March 2008 without complying with the applied treatments. The 120 patients were randomly divided into 4 groups and were provided, by lot and in a blinded fashion, with four different treatment cycles consisting of the following drugs: Group A $(\mathrm{n}=20) 600 \mathrm{mg} /$ day of alpha lipoic acid for two months, Group B $(\mathrm{n}=20) 300 \mathrm{mg} /$ day of gabapentin for two months, Group C ( $\mathrm{n}=20)$ a combination of both drugs for two months and Group D ( $\mathrm{n}=60) 100 \mathrm{mg} /$ day of cellulose starch for two months (control group).

Results and Conclusions: All 120 patients completed the treatment. The best response was obtained with the combination of ALA + GABA, with a $70 \%$ of the cases with reduced burning in this group and a 13.2 times greater chance of presenting positive changes for these patients than those taking placebo. The combined use of drugs that act at different levels of the nociceptive system can be useful for the treatment of this syndrome.
\end{abstract}

Key words: Burning mouth syndrome, pharmacological treatment of burning mouth, alpha lipoic acid, gabapentin, oral neuropathic pain. 


\section{Introduction}

Burning mouth syndrome (BMS) is a disease characterized by the presence of burning mouth, taste disturbances and dry mouth sensation in the absence of injuries that justify it (1-3). The sensation of "burning" generally involves the tongue, at its tip and edges, and can be extended to any area of the mouth. It often occurs more frequently in women over 60 years in the peri-or postmenopausal period; it is rarely found in men $(1,2)$. The etiology is unknown but it's believed to be multifactorial; patients with BMS often have anxiety and clinical depression $(4,5)$. Among the important local factors dental trauma, the presence of tartar, rough surfaces prosthesis, allergic reactions to dental materials, chemical irritants of lipsticks, toothpastes and prolonged use of mouthwashes may be included. Among the general factors deficiency states, deficiencies of iron, folic acid and vitamin B are important (6-9). Alpha Lipoic Acid is a component produced in the body in very small quantities, being present in many foods such as spinach, broccoli and meats. This substance acts as a coenzyme in various reactions, taking part in glycolysis - responsible for the conversion of blood sugar into energy- and in the regeneration of liver tissue damage. It also exerts a neuro-regenerative action, because it crosses the hematoencephalic barrier and has a protective effect of brain and nerve tissue $(10,11)$. A systematic review of the Cochrane Collaboration, noted that Alpha-Lipoic Acid (ALA) may help in management of burning mouth syndrome (12). Various studies have shown that the use of ALA did not produce greater benefits on the burning mouth that the use of placebos $(13,14)$. Gabapentin is an anticonvulsant whose chemical structure consists of a molecule of gamma-aminobutyric acid covalently linked to a lipophilic cyclohexane ring. This drug was created to act as an agonist of the inhibitory neurotransmitter GABA of central activity since it is easily transferred to the central nervous system (CNS) for having high lipid solubility. The mechanism of action of this drug is still unknown because it acts by increasing the discharge promoted of GABA but without noting a steady reduce in the potentials of action or affection of the calcium channel current (15). The objective of this research was to test the action of alpha lipoic acid and gabapentin alone and jointly, on the symptoms of BMS.

\section{Patients and Methods}

We conducted a randomized clinical double-blind, placebo-controlled clinical trial among patients with BMS seen at the Chair of Clinic Stomatology II of the Faculty of Dentistry, Rosario, Argentina. The study was approved by the Bioethics Committee of the Institution. Sample size was determined by considering a confidence level of $95 \%$ and an error rate of $+/-5 \%$, determining the need to include a number of 120 patients for our study.
The study was conducted during April and May 2008. The 120 patients were selected among those patients with BMS who have been treated at our service between March 2003 and March 2008, without responding to the applied treatments. A total of 120 patients with idiopathic BMS of more than three months duration that wanted to participate voluntarily were included. Polypharmacy patients using more than 3 systemically daily drugs, those ones taking psychotropic and antihypertensives drugs as well as patients with serious psychiatric conditions previously diagnosed were excluded. Patients with deficiencies of folic acid, vitamin B, carriers of anemias of any kind and patients with Sjögren syndrome were also excluded. Participants were warned of the possibility of access to different drugs as well as the possibility of being treated with placebo. An informed consent was signed where the scope of the study and the freedom to leave treatment at any time were specified, and also the provision of medication following the completion of the study was ensured. We performed an initial psychological diagnosis for all patients through the application of the Hospital Anxiety and Depression Scale (HAD) and the Hamilton Rating Scale for Depression in order to meet the psychological situation of our patients. Then we evaluated the presence of burning through a numerical scale especially created for this work, describing the burning from 0 to 4 , where the 0 value corresponded to the absence of burning, the 1 value to the presence of burning in a single area of the tongue, the 2 value to two distinctive areas (tongue and gums, tongue and lips or tongue and palate), the 3 value to three areas and the 4 value corresponded to burning spread throughout the mouth. This specific designed scale, which considered the geographical distribution of burning in different areas of the mouth, allowed us to distinguish improvements or deteriorations of burning sensation in the various assessments. The 120 patients were randomly divided into 6 groups of 20 members each. Once the groups were formed, our service attendants assigned a numerical order for each group being identified as groups $1,2,3,4,5$ and 6 . On the other hand, six treatment cycles were determined: cycles A, B, C, D, E and $\mathrm{F}$, so that cycle $\mathrm{A}(\mathrm{n}=20)$ corresponded to $600 \mathrm{mg} /$ day of ALA, the cycle B $(n=20) 300 \mathrm{mg} /$ day of GABA, the cycle $C(n=20)$ to the combination of both drugs and the cycles $D(n=20), E(n=20)$ and $F(n=20)$ were $100 \mathrm{mg}$ / day of starch and cellulose (Placebo). The support staff of our service made a draw with 6 balls to link the groups with the cycles of treatment. After the draw, the three groups were combined with cycles $\mathrm{D}, \mathrm{E}$ and $\mathrm{F}$ to be treated with placebo, thus forming a single group for these patients, Group $\mathrm{D}(\mathrm{n}=60)$ or control group proceeding to medication delivery, allocation that was always masked to both patients and researcher, through the use of capsules of similar size and appearance so that just the support staff was the one who recorded the information until 
the end of the treatment (blind). The beginning of the administration of the different drugs was concomitant for all patients, as well as the evaluation of the effects, which was made monthly and also blinded (double blind), always by the same researcher using the protocol described above, which was completed for the first time the day before the start of treatment and thirty and sixty days, respectively. To evaluate the changes that occurred with the taking of the different drugs, it was established that the improvements (positive changes) involved the passage of a certain level or numerical category of burning to a lower one, the deteriorations (negative changes) involved an increase of a certain level of burning to a higher one and the total resolution indicated the total absence of burning, that is to say the transition from any higher value to zero value. In this way four categories were obtained for the analysis of the results: Category 1: with negative changes (deterioration), Category 2: no changes; Category 3: with positive changes (improvements) and Category 4: with total recovery. The Chi- square test was used for the analysis to an independent variable with a predictive value $(\mathrm{P}$ $<0.05)$ and the Odds ratios were calculated to determine the relationship between treatment and its effect on the intensity of the burning with ( $\mathrm{P}<0.01)$. All subjects were evaluated through an Intention-to-Treat Analysis which would take into account all patients although they could discontinue the treatment.

\section{Results}

All patients included initially ended the study because the duration of treatment lasted only 2 months and the adverse effects that appeared were very mild. Of the 120 patients studied, 94 were females and 26 males with a mean age of $14.1 \pm 57.5$ years, median: 57 . The psy- chological diagnosis showed 95 patients without pathology $(78 \%), 14$ with anxiety $(13 \%)$ and 11 with depression (9\%). We assessed changes in levels of burning in each group after one and two months of treatment. The first month of treatment, changes in levels of burning were not dependent on the type of drug tested ( $p$-value $=0.293$ ) (Fig. 1). After 2 months of treatment, we found that the positive changes in the levels of burning, significantly depended on the type of drug assigned ( $p$-value $<0.001$ ) (Fig. 2). When relating the changes in patients treated with ALA, GABA or the combination, and comparing them with changes in the group of patients who received placebo, we observed the existence of a large percentage of patients with negative changes within this group (Fig. 3). When observing the patients who received ALA, we found that 9/20 (45\%) of these patients did not presented changes, while the remaining 11/20 (55\%) had positive changes or full recovery in the case. Among the patients treated with GABA, 10/20 (50\%) remained unchanged while the rest manifested decrease in the burning. Among those who were given the combination of both drugs, only 6/20 (30\%) remained unchanged while 14/20 (70\%) had positive changes. Finally, and within the group of patients receiving placebo, $51 / 60(85 \%)$ remained unchanged or worsened while $9 / 60(15 \%)$ had positive changes. The calculation of odds ratios determined that the possibility of presenting positive changes (or total resolution of the case) for patients treated with ALA was 7 times higher than for those receiving placebo. For those patients who received GABA, that possibility was 5.7 times higher than for the control group and for the patients who received ALA and GABA together, that possibility was 13.2 times higher than for the control patients group (Table 1).

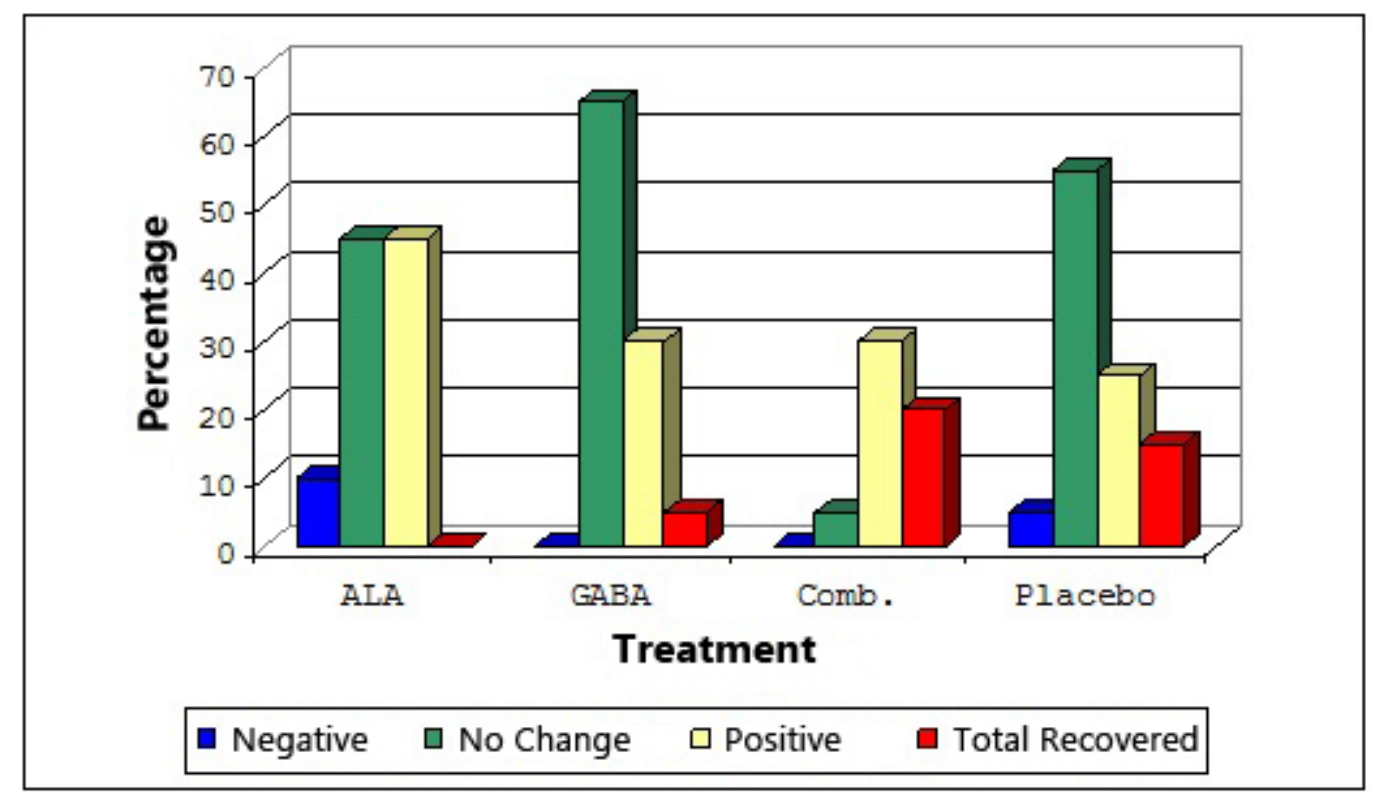

Fig. 1. Analysis of the changes after one month of treatment. 


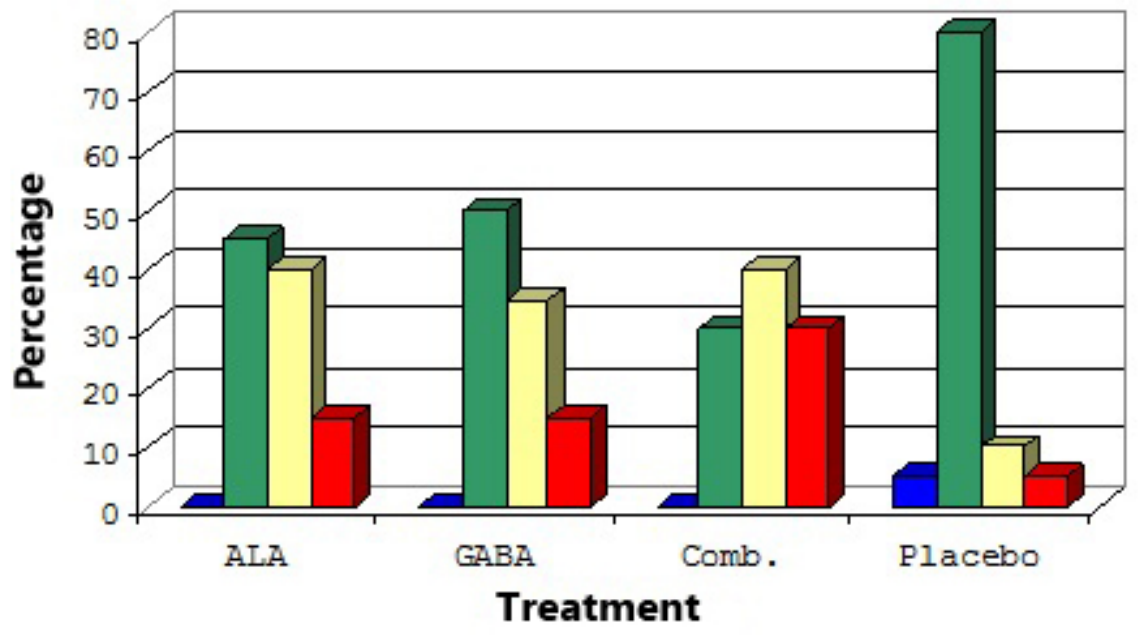

- Negative $\square$ No Change $\square$ Positive $\square$ Total Recovered

Fig. 2. Analysis of the changes after two months of treatment.

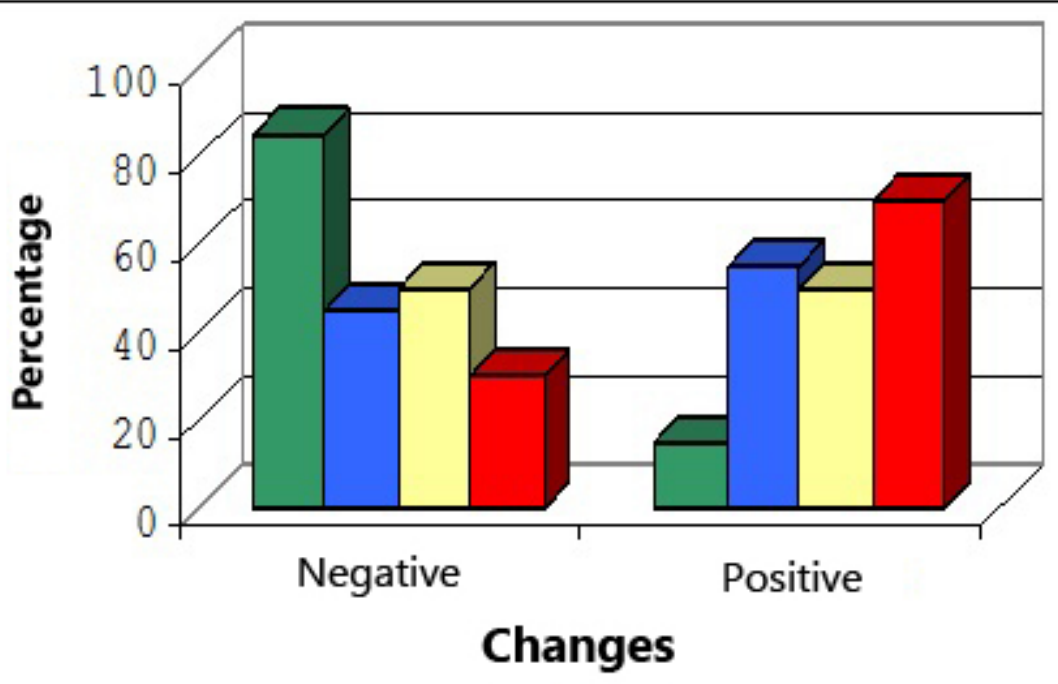

\section{$\square$ Placebo $\square$ ALA $\square$ GABA $\square$ Comb.}

Fig. 3. Comparison of the changes caused by the different drugs after two months.

Table 1. Favorable changes after two months of treatment

\begin{tabular}{|c|c|c|}
\hline Treatment & $\begin{array}{c}\text { Percentage of } \\
\text { Favorable Cases \# }\end{array}$ & $\begin{array}{c}\text { Possibility of } \\
\text { Favorable Results * }\end{array}$ \\
\hline $\begin{array}{c}\text { Alpha lipoic Acid } \\
\text { (ALA) }\end{array}$ & $55 \%$ & 7 times better \\
\hline $\begin{array}{c}\text { Gabapentin } \\
\text { (GABA) }\end{array}$ & $50 \%$ & 5.7 times better \\
\hline $\begin{array}{c}\text { Combination } \\
\text { (ALA+GABA) }\end{array}$ & $70 \%$ & 13.2 times better \\
\hline
\end{tabular}

\# Includes Patients with total case resolution.

$*=(\mathrm{p}$ value $<0,001)$ compared with the placebo group. 


\section{Discussion}

The communication of different therapeutic measures to control the BMS is wide in the world literature (1120). The Gabapentin was used by White et al. (15) in a case of a patient with BMS obtaining healing of the case and it was subsequently used by Heckmann et al. (16) who conducted a study with gabapentin in 15 patients, where the initial dose was $300 \mathrm{mg}$ / day and then grew at a rate of $300 \mathrm{mg}$ every 48 hours to a maximum of $2400 \mathrm{mg} /$ day. Seven of the patients were treated for two weeks, 6 patients for four weeks and another 2 patients for six weeks. Although 2 patients (13\%) indicated a decrease in pain of three points and another 2 patients (13\%) showed a decrease of two points on the VAS (visual analogue scale), the authors concluded that gabapentin has little or no effect on the treatment of BMS. In our study, we used GABA individually, with fixed doses of $300 \mathrm{mg}$ daily for two months, getting no major differences with the published studies, although obtaining improvements when used it in combination with ALA. Femiano et al. (17) used the ALA at a dose of $600 \mathrm{mg} /$ day and psychotherapy, in a parallel group and placebo-controlled trial. This drug was chosen for its neuroregenerative properties, demonstrated in the treatment of diabetic neuropathy, another disease with similar characteristics to the BMS that causes chronic pain. In the group treated with a total of 48 patients, 5 patients (10\%) experienced a slight improvement, 19 patients $(40 \%)$ a clear improvement and 15 patients $(31 \%)$ recovered from the BMS. The $81 \%$ of patients treated with the combination of ALA + Psychotherapy experienced some improvement. In the placebo group only 6 patients (13\%) experienced improvement, and this one was classified as mild. At six months 19 patients (49\%) who had experienced improvement after two months of treatment with ALA worsened. The same group has published other studies with ALA under different conditions but with similar results. Lopez Jornet et al. (20) also used ALA in 30 patients but at a concentration of $800 \mathrm{mg}$ daily for eight weeks with no statistically significant differences when comparing the treatment group with the control group. Carbone et al. (12) conducted a randomized, double-blind placebo-controlled study among 54 women and 12 men who used $400 \mathrm{mg}$ of ALA daily together with vitamins, forming three groups administered, for 16 weeks, with $400 \mathrm{mg}$ / day of ALA, $400 \mathrm{mg} /$ day of ALA + vitamins and Placebo, getting similar effects in all three groups demonstrating a lack of efficacy of ALA in BMS, as this did not produce better effects than placebo. In a review of a series of 31 patients with BMS who took $600 \mathrm{mg} /$ day in divided doses of ALA, Steele et al. (13) determine that the drug was beneficial in only 11 patients of all patients surveyed. Cavalcanti and da Silveira (14) performed a randomized double-blind, placebo-controlled trial between
48 patients divided into two treatment cycles alternating 20-day $200 \mathrm{mg} /$ day of ALA and $100 \mathrm{mg} /$ day of cellulose starch, but were not able to demonstrate the greater efficacy of ALA over placebo. In our experience with the administration of ALA in 20 patients, with a daily dose of $600 \mathrm{mg}$ for two months, we obtained similar results to those of Femiano et al. (17), with a decrease of the burning sensation in $55 \%$ of patients treated, being the possibility of favorable changes 7 times higher for patients who received ALA than for the control group. Note that in our study we also indicated as a treatment for one group the combination of ALA + GABA to try to produce an action on both peripheral and central nociceptive system, obtaining with this combination the best results. Although effective treatments have been exposed to particular cases, we are still looking for a drug therapy that could be effective for most patients with BMS.

\section{Conclusions}

Gabapentin and Alpha Lipoic Acid used both individually and jointly, were beneficial for reducing the burning in patients with BMS. The most favorable results were obtained with the administration of both drugs. To establish specific therapies in the treatment of BMS, further study of the pathophysiological mechanisms involved in its pathogenesis would be required, as well as determine the neurological changes involved in this disease, focusing on this entity within the Central Sensitization Syndromes. Given the limitations in time and in the number of patients in our study, we recommend conducting multicenter clinical trials in more prolonged treatments, based on the combined use of drugs acting at different levels of the nociceptive system, since that could be the key to future advances in the treatment of this disease.

\section{References}

References with links to Crossref - DOI

1. Palacios-Sánchez MF, Jordana-Comín X, García-Sívoli CE. Burning mouth syndrome: a retrospective study of 140 cases in a sample of Catalan population. Med Oral Patol Oral Cir Bucal. 2005;10:38893.

2. Silvestre FJ, Serrano C. Burning mouth syndrome: concepts review and update. Med Oral. 1997;2:30-38.

3. Bergdahl J, Anneroth G. Burning mouth syndrome: literature review and model for research and management. J Oral Pathol Med. 1993;22:433-8.

4. Bogetto F, Maina G, Ferro G, Carbone M, Gandolfo S. Psychiatric comorbidity in patients with burning mouth syndrome. Psychosom Med. 1998;60:378-85.

5. Basker RM, Main DM. The cause and management of burning mouth condition. Spec Care Dentist. 1991;11:89-96.

6. Chimenos-Kustner E, Marques-Soares MS. Burning mouth and saliva. Med Oral. 2002;7:244-53.

7. Bergdahl J. Psychologic aspects of patients with symptoms presumed to be caused by electricity or visual display units. Acta Odontol Scand. 1995;53:304-10.

8. Miyamoto SA, Ziccardi VB. Burning mouth syndrome. Mt Sinai J Med. 1998;65:343-7. 
9. Bergdahl M, Bergdahl J. Burning mouth syndrome: prevalence and associated factors. J Oral Pathol Med. 1999;28:350-4.

10. Lauritano D, Spadari F, Formaglio F, Zambellini Artini M, Salvato A. Etiopathogenic, clinical-diagnostic and therapeutic aspects of the burning mouth syndrome. Research and treatment protocols in a patient group. Minerva Stomatol. 1998;47:239-51.

11. Femiano F, Gombos F, Scully C, Busciolano M, De Luca P. Burning mouth syndrome (BMS): controlled open trial of the efficacy of alpha-lipoic acid (thioctic acid) on symptomatology. Oral Dis. 2000;6:274-7

12. Carbone M, Pentenero M, Carrozzo M, Ippolito A, Gandolfo S. Lack of efficacy of alpha-lipoic acid in burning mouth syndrome: a double-blind, randomized, placebo-controlled study. Eur J Pain. 2009;13:492-6.

13. Steele JC, Bruce AJ, Drage LA, Rogers RS 3rd. Alpha-lipoic acid treatment of 31 patients with sore, burning mouth. Oral Dis. 2008;14:529-32.

14. Cavalcanti DR, da Silveira FR. Alpha lipoic acid in burning mouth syndrome--a randomized double-blind placebo-controlled trial. J Oral Pathol Med. 2009;38:254-61.

15. White TL, Kent PF, Kurtz DB, Emko P. Effectiveness of gabapentin for treatment of burning mouth syndrome. Arch Otolaryngol Head Neck Surg. 2004;130:786-8.

16. Heckmann SM, Heckmann JG, Ungethüm A, Hujoel P, Hummel T. Gabapentin has little or no effect in the treatment of burning mouth syndrome - results of an open-label pilot study. Eur J Neurol. 2006;13:e6-7.

17. Femiano F, Gombos F, Scully C. Burning Mouth Syndrome: open trial of psychotherapy alone, medication with alpha-lipoic acid (thioctic acid), and combination therapy. Med Oral. 2004;9:8-13.

18. Maina G, Vitalucci A, Gandolfo S, Bogetto F. Comparative efficacy of SSRIs and amisulpride in burning mouth syndrome: a singleblind study. J Clin Psychiatry. 2002;63:38-43.

19. Petruzzi M, Lauritano D, De Benedittis M, Baldoni M, Serpico R. Systemic capsaicin for burning mouth syndrome: short-term results of a pilot study. J Oral Pathol Med. 2004;33:111-4.

20. López-Jornet P, Camacho-Alonso F, Leon-Espinosa S. Efficacy of alpha lipoic acid in burning mouth syndrome: a randomized, placebo-treatment study. J Oral Rehabil. 2009;36:52-7. 